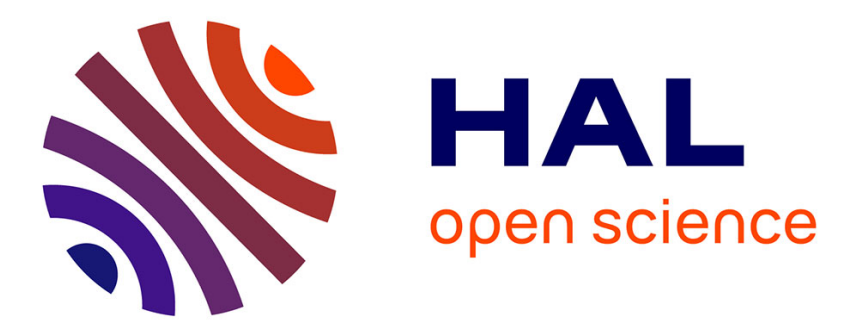

\title{
Advances in polymer-based optical MEMS fabrication for VCSEL beam shaping
}

Véronique Bardinal, Thierry Camps, Benjamin Reig, Sami Abada, Emmanuelle Daran, Jean-Baptiste Doucet

\section{To cite this version:}

Véronique Bardinal, Thierry Camps, Benjamin Reig, Sami Abada, Emmanuelle Daran, et al.. Advances in polymer-based optical MEMS fabrication for VCSEL beam shaping. IEEE Journal of Selected Topics in Quantum Electronics, 2014, 21 (4), pp.Article number 2700308. 10.1109/JSTQE.2014.2369743 . hal-01158903

\section{HAL Id: hal-01158903 \\ https://hal.science/hal-01158903}

Submitted on 2 Jun 2015

HAL is a multi-disciplinary open access archive for the deposit and dissemination of scientific research documents, whether they are published or not. The documents may come from teaching and research institutions in France or abroad, or from public or private research centers.
L'archive ouverte pluridisciplinaire HAL, est destinée au dépôt et à la diffusion de documents scientifiques de niveau recherche, publiés ou non, émanant des établissements d'enseignement et de recherche français ou étrangers, des laboratoires publics ou privés. 


\title{
Advances in polymer-based optical MEMS fabrication for VCSEL beam shaping
}

\author{
V. Bardinal, Member, IEEE, T. Camps, B. Reig, S. Abada, E. Daran and J. B. Doucet
}

\begin{abstract}
In this paper, we discuss the design, fabrication, and characterization of electro-thermally-actuated polymer-based micro-optical electro-mechanical microsystems (MOEMS) for active microoptics in VCSEL devices. We describe in particular the principle of a SU8-based MOEMS designed for single-mode VCSEL beam active focusing. The ultimate objective is the realization of parallel compact optical scanners for sensing applications using collective and low-cost technologies. After discussing the advantages of the epoxy resist SU-8 for fabricating an integrated movable lens on active optical devices, we present our latest advances in technology for ensuring precise MOEMS fabrication on small III-V samples and for achieving accurate alignment of lenses on suspended circular membranes. Finally, we present our first results on the beam focusing of multimode VCSELs, which demonstrate the feasibility of our approach and could provide new insights in the MEMS-VCSEL field.
\end{abstract}

Index Terms-VCSEL, MEMS, MOEMS, polymer, microoptics, SU-8, microlens fabrication, self-alignment, collective fabrication, electro-thermal actuation.

\section{INTRODUCTION}

$\mathrm{V}$ CSELs (vertical cavity surface emitting lasers) are currently the first choice in photonic devices for an increasing range of applications, including short-distance data

Submission date: 30 September 2014.

This work was partially supported by RENATECH (French Network of Major Technology Centers) within LAAS-CNRS for technological support and Region Midi-Pyrénées (FIAB SU-8 project). The French National Research Agency (ANR) is also gratefully acknowledged for partial financial support (ANR-09-BLAN-0168-01).

V. Bardinal, B. Reig, S. Abada, E. Daran and J.B. Doucet are with CNRS, LAAS (Laboratory of Analysis and Architecture of Systems of French National Scientific Research Center), 7 avenue du colonel Roche, F-31400 Toulouse, France and with Univ de Toulouse, LAAS ; F-31400 Toulouse, France. (e-mail: bardinal@laas.fr).

T. Camps is with CNRS, LAAS (Laboratory of Analysis and Architecture of Systems of French National Scientific Research Center), 7 avenue du colonel Roche, F-31400 Toulouse, France and Univ de Toulouse, UPS, LAAS, F-31400 Toulouse, France (e-mail: camps@laas.fr). communications, optical sensors and laser printers [1-3], as well as flow cytometry [4-5], parallel optical trapping [6], nano-bio-sensing [7] and optical probe microscopy [8]. This success is due to the devices' outstanding characteristics, such as parallel operation, low threshold current, high modulation rate and a circularly symmetric emitted beam. Over the past 30 years, the development of VCSELs has been directly linked to major technological breakthroughs, such as the achievement of high-quality epitaxial growth of quantum wells in the emitting zone [9], current injection in doped distributed Bragg reflectors (DBR) [9][10], improvements in efficiency coupled to transverse mode control using buried lateral oxidation (AlOx) [11], buried tunnel junction insertion (BTJ) [12], and the exploitation of new optical effects due to advancements in nanophotonics [13-14]. Additional functionalities have also been reported in coupling VCSELs to MEMS (micro-electromechanical systems) in two main configurations. The best known configuration involves the spectral tuning of laser emission for WDM or spectroscopy applications [15]. It consists in creating an intra-cavity air gap between a highly reflective suspended membrane or cantilever and a halfVCSEL structure. The air gap is varied under MEMS actuation, which allows for the wavelength of the laser's emitted light to be tuned. The first demonstrations of tunable MEMS-VCSELs were fully monolithic; they were based on the insertion of a III-V sacrificial layer in the VCSEL multilayer structure (air gap thickness of $\sim 1 \mu \mathrm{m}$ ) [16-17]. Other approaches relying on the hybrid assembly of a movable dielectric mirror were also reported [18-21]. With this configuration, wider spectral tuning ranges are achievable owing to the large stop band of the top mirror.

The other MEMS-VCSEL configuration features microoptics for laser beam collimation, beam focusing and/or beam deflection. The natural beam divergence of a standard single-frequency VCSEL is indeed too large to make its direct use possible in systems without any correction lenses. Realtime control of beam position during laser operation is also a key issue in incorporating VCSELs into compact systems, whether for optical communications (reconfigurable optical routers) or for instrumentation or biomedical analysis (optical scanners) [22]. Consequently, dynamic control of lens displacement constitutes a significant advantage. These features are generally provided by bulky optical elements. To address these issues in a more compact manner, a MEMS featuring a movable microlens (refractive or diffractive) has to be placed at a distance on the order of its focal length from the 
VCSEL surface (typically few tens of microns to few hundreds of microns). As a result, monolithic MEMS fabrication is no longer viable. Most proposed solutions are based on the hybrid assembly of silicon-based MOEMS and are aimed at beam steering applications (microlens off-axis lateral displacement) [23-29]. However, the design of such MOEMS (micro-optical electro-mechanical microsystems) with dimensions compatible with the pitch of VCSEL arrays remains critical. Furthermore, direct integration should be preferred to avoid tricky assembly steps between the sources and the micro-lenses. To this end, the authors recently reported a new type of polymer-based MOEMS that can be directly and collectively integrated on VCSEL wafers [30-31]. In this paper, we detail the advantages of our approach with respect to post-process fabrication and wafer-scale integration capabilities. We also present our latest advances in MOEMS technology in attaining more uniform and self-aligned lens fabrication. Finally, we discuss our latest experimental results regarding MOEMS performance and the potential these devices hold.

\section{INTEREST IN POLYMER MOEMS FOR MICROOPTICS APPLICATIONS ON VCSELS}

Polymer-based technologies present numerous advantages over technologies based on inorganic materials with respect to micro/nano-fabrication. Specifically, polymer-based technologies usually involve simple, low-cost and lowtemperature fabrication steps $\left(<200^{\circ} \mathrm{C}\right)$ that are compatible with the post-processing of any functional device. Among such technologies, those based on photosensitive resists allow for great flexibility in pattern design due to photolithography. In particular, thick resists such as SU-8 make the fabrication of high-aspect-ratio patterns with good sidewall quality and mechanical stability possible [32-33]. As a result, this negative-tone epoxy photoresist has been widely used by the MEMS community, initially for micro-mold fabrication for electroplating or as a master material for hot embossing but also as a structural material [34-37]. This resist is of particular interest for fabricating electro-thermally actuated MEMS because it presents a low thermal conductivity $\left(0.2 \mathrm{Wm}^{-1} \mathrm{~K}^{-1}\right)$ combined with a high thermal expansion coefficient (52 $\mathrm{ppm} /{ }^{\circ} \mathrm{C}$ ), enabling large deformations at low applied power and no detrimental substrate heating. These properties must be compared to those associated with silicon (see Table I). Moreover, the initial thermo-mechanical stress in SU-8 is lower than that in Si-based materials. Finally, the Young's modulus of SU-8 is quite high for a polymer material, making large elastic deformations possible.

TABLE 1: COMPARISON OF SI AND SU-8 PROPERTIES AS A STRUCTURAL MATERIAL FOR ELECTRO-THERMAL MEMS FABRICATION

\begin{tabular}{|c|c|c|}
\hline Material & $\mathbf{S i}$ & $\mathbf{S U - 8}$ \\
\hline $\begin{array}{c}\text { Thermal conductivity } \\
\mathbf{K}_{\text {th }}\left(\mathbf{W ~ m}^{-1} \mathbf{K}^{-1}\right)\end{array}$ & 149 & 0.2 \\
\hline $\begin{array}{c}\text { Linear thermal expansion } \\
\text { CTE }\left(\mathbf{1 0}^{-6} \mathbf{K}^{-1}\right)\end{array}$ & 2.6 & 52 \\
\hline Young's modulus (GPa) & 165 & 4 \\
\hline
\end{tabular}

Compared to silicon, one disadvantage should be noted: a lower reliability that must still be studied and improved [38]. We recently reported an assessment of the reliability of SU-8 microstructures for passive micro-optics [39] with the aim of extending this study on movable structures. Nevertheless, SU8 presents specific advantages for MOEMS due to its optical properties. SU-8 is indeed transparent over the visible and near infra-red ranges with absorption losses lower than 3 $\mathrm{dB} / \mathrm{cm}$ [40]. SU-8 has been fruitfully exploited for the fabrication of organic waveguides, micro-resonators [40] and microlens arrays [41]. Additionally, the integration of SU-8based microlenses on VCSELs has been reported, taking advantage of the properties of SU-8 cylindrical pedestals [4344]. These authors used inkjet printing to deposit curable microlens material on SU-8 pedestals and achieved in this manner precise control of lens dimensions owing to liquid self-positioning. More recently, we exploited similar selfcentering properties using an alternative low-volume deposition technique based on a robotized silicon-cantilever spotter [45]. Moreover, we showed that VCSEL beam divergence can be tailored by properly controlled SU-8 pedestal dimensions by photolithography. Taking advantage of all these properties, we also reported on the fabrication of a SU-8-based MOEMS suitable for VCSEL dynamic beam control. The tunable lens we propose is composed of a thermally actuated SU-8 membrane surmounted by a polymer microlens (fig. 1). This membrane can be shifted vertically under an applied thermal gradient due to thermal expansion. Actuation of the microlens's position produces an axial shift in the laser beam's waist.

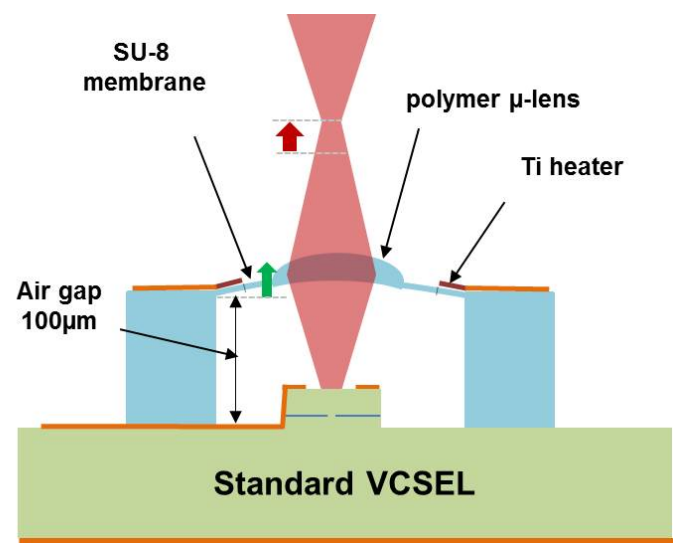

Figure 1: Schematic view of the SU-8 optical MEMS on a standard VCSEL for dynamic focusing.

MOEMS dimensions such as the air gap size, membrane thickness and membrane diameter were optimized using an optical modeling tool, ZEMAX-EE, taking into account technological capabilities and compatibility with final integration on single-mode VCSELs emitting at $850 \mathrm{~nm}$ [46]. The results of this study show that above a minimal air gap value $(>100 \mu \mathrm{m})$, the VCSEL beam can be focused at a sufficient working distance $(>300 \mu \mathrm{m})$ to allow for the combination of such lensed VCSEL and a microfluidic channel in a compact optical sensor. Moreover, a $5 \mu \mathrm{m}$ axial shift induces a significant change in the axial position of the beam waist (several tens of micrometers) while maintaining a 
beam waist size smaller than $10 \mu \mathrm{m}$ such that a complete scan of a microfluidic channel can be performed, with a resolution that cannot be achieved using bulk optics [47]. Compared to a hybrid assembly of Si-based MOEMS, the major interest in our approach lies in collective fabrication based on photolithography. In this manner, it is possible to comply with the standard pitch of individual VCSELs in an array $(250 \mu \mathrm{m}$ or $500 \mu \mathrm{m})$ and with lateral tolerances to optical alignments because the precision of photolithography is better than $2 \mu \mathrm{m}$.

\section{SU-8 MOEMS FABRICATION ABOVE VCSELS}

The simple method we developed for MOEMS integration on VCSELs is based on the double exposure of SU-8, first proposed by Ceyssens and Puers for the fabrication of microfluidic channels [48]. After VCSEL fabrication (fig. 2a), a single thick SU-8 layer is deposited by standard spin-coating (fig. 2b). This layer is then exposed to two successive UV exposure steps through two different photolithography masks. The first is realized at the typical wavelength, $\lambda_{1}=365 \mathrm{~nm}$, and leads to the complete exposure of the MOEMS's lateral anchors (fig. 2c). The second is performed at a shorter wavelength, $\lambda_{2}=320 \mathrm{~nm}$, using a filter placed in the optical path, which results in the definition of a thin membrane measuring $5 \mu \mathrm{m}$ in thickness (fig. 2d). A standard development process is then applied to remove the uncrosslinked SU-8 and release the membrane (fig. 2e). After a standard SU-8 hard bake, a thin film of titanium is deposited and locally patterned to create a resistive heating electrode on the membrane edge (fig. 2f). Finally, a microlens is formed by local depositing a curable liquid droplet at the center of the circular membrane (fig.2 g). The main advantage of this method is avoiding the use of a sacrificial layer composed of another material for membrane definition and release. To make this fabrication method fully compatible above VCSEL devices, we had to resolve two issues: the uniformity of the SU-8 thickness on small III-V samples and precise control of lens positioning on the membrane. The following sections describe the generic solutions we recently developed to resolve these issues.

\section{A. Improvement in SU-8 thickness uniformity using soft thermal printing}

For a targeted thickness of $100 \mu \mathrm{m}, \mathrm{SU}-8$ is highly viscous and suffers from a lack of thickness uniformity after spincoating, particularly if the wafer on which it is deposited is not circular. This configuration generates annular beads from the edge of the wafer that become highly detrimental in the case of small-sized samples. For the precise and uniform fabrication of a $100-\mu \mathrm{m}$-thick SU-8 MOEMS on small-sized or non-circular samples such as III-V samples (fig. $2 b$ ), we recently developed an alternative method to SU-8 spin coating that we call "soft thermal printing". The method is derived from the SU-8 dry film lamination process [49] and based on the use of a set-up equipped with a double membrane system initially devoted to nano-imprint lithography (NIL). Due to the combination of applied hydrostatic pressure and uniform heating, this method allows for the soft transfer of a uniform, un-crosslinked SU-8 dry film (previously deposited on a flexible sheet) on a sample. After a final photolithography step, the uniformity of fabricated SU-8 patterns was observed to have been improved from $30 \%$ to $5 \%$ over 2 -cm square samples, with a $5 \mu \mathrm{m}$ maximal deviation from the target value (fig. 3). Compared to film lamination, this method is applicable regardless of sample fragility. More details can be found elsewhere [50].

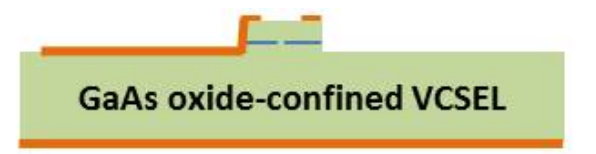

a) VCSEL standard fabrication.

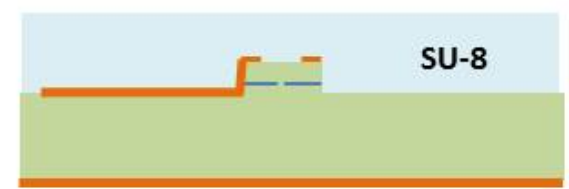

b) thick SU-8 spin coating or soft printing. Soft-bake.

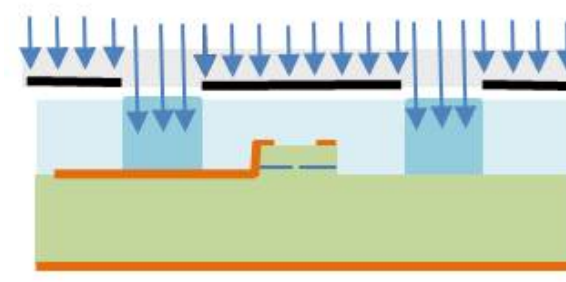

c) first exposure. $\lambda_{1}$

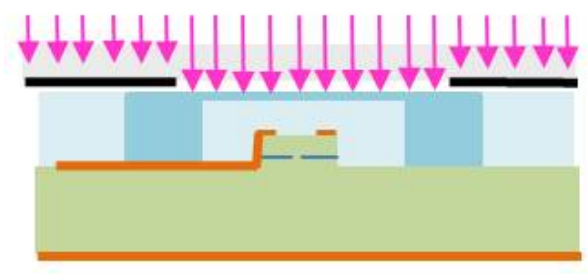

d) second exposure. $\lambda_{2}$

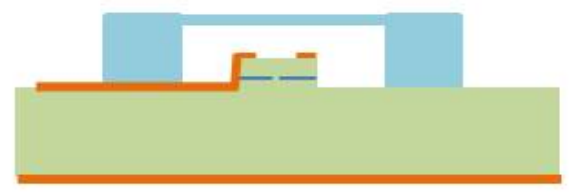

e) SU-8 developing. Hard-Bake.

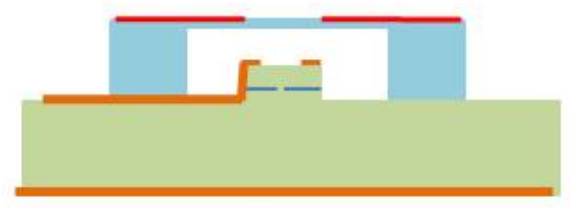

f) Ti electrode deposition and etching.

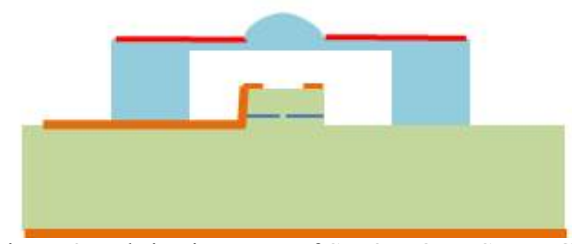

g) polymer $\mu$-lens deposition. 


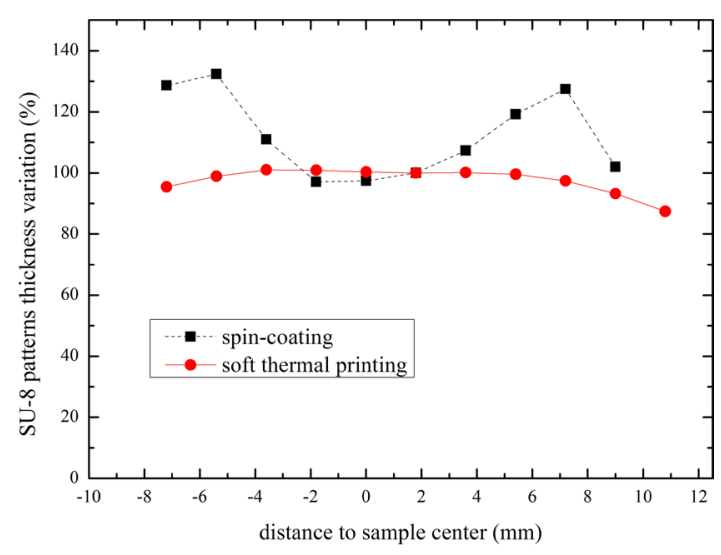

Figure 3: Normalized thickness profile of SU-8 micro-patterns fabricated by photolithography after a standard spin-coating (black squares, dotted line) and after soft-thermal printing (red circles, straight line) on a 2-cm square sample $[50]$.

\section{B. Self-aligned microlenses on suspended membranes}

Lens deposition is a critical stage of MOEMS fabrication (fig. 2g). We used a liquid dispensing technique because it is already proven for passive microoptics on VCSELs [44][45] and enables high lens surface quality and compatibility with a non-planar process. However, for deposition on a suspended membrane, liquid droplets cannot be fully delimited by the boundaries as they are on a bulk SU-8 pedestal. Nevertheless, self-alignment on a photolithographic pattern remains, in our opinion, the best way for precise and truly collective fabrication. Therefore, we included in our membrane design a centering notch close to each actuation arm to prevent liquid from spreading towards the arms after deposition (fig. 4). These holes were created during the second UV exposure, at the same time the thin membrane was formed (fig. 2d), by taking advantages of proximity effects occurring inside the micrometer-scale patterns [51]. Therefore, no additional step was necessary. Two different local liquid dispensing methods were exploited for lens fabrication on these MOEMS: robotized micro-spotting and inkjet printing. In the following paragraphs, we discuss the respective advantages of both techniques, especially in terms of lens curvature radius control.

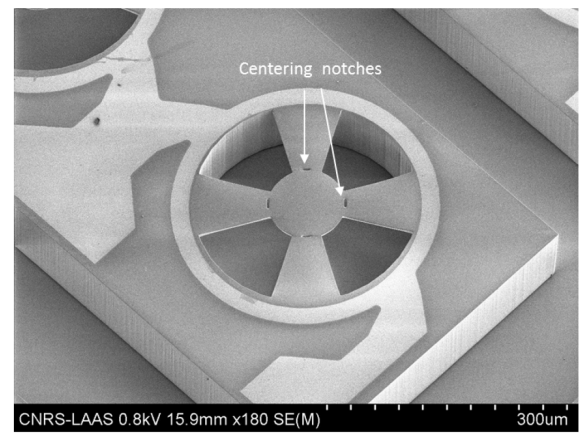

Figure 4: SEM image of a MEMS with 4 actuation arms and centering notches for a better liquid self-centering (circular membrane diameter: $100 \mu \mathrm{m}$ ).

\section{1) Lens deposition using a robotized micro-spotter}

The authors previously reported a low-cost and simple technique based on a silicon micro-cantilever spotter for high- quality polymer microlens fabrication [52]. By combining this contact technique with the use of SU-8 cylindrical pedestals, the authors demonstrated accurate lens self-centering and VCSEL collimation [45]. The principle is as follows: a lowviscosity liquid is loaded by capillary forces into a cantilever channel and droplet deposition is achieved by placing the cantilever in contact with the sample surface (fig. 5a). The main advantage of this contact technique lies in its simplicity: indeed, it is limited only ruled by natural surface energy. The dispended volume remains constant regardless the contact time (beyond a minimal value). For a given surface and a given polymer, the focal length of the lens relies solely on its diameter. We tested this method for lens deposition on a membrane with a diameter of $100 \mu \mathrm{m}$ using two different lowviscosity materials having optical properties similar to those of SU-8. The first one was a UV-curable epoxy-polymer (DMSE) with a natural contact angle of $29^{\circ}$ on SU-8. However, the surface wettability of SU-8 was observed to strongly increase after chemical etchings steps during MOEMS fabrication (contact angle $<10^{\circ}$ ). As a result, we noted extensive liquid spreading on the arms (fig. 5b), even in the presence of centering notches. We therefore had to apply a wet chemical treatment that rendered the surface more "hydrophobic" to recover a higher contact angle $\left(32^{\circ}\right)$ (fig. 6a). We also deposited a thermo-curable material exhibiting a higher contact angle on the same treated surface (polyester, $46^{\circ}$ ) (fig. 6b). In both cases, the microlens centered on the circular membrane was observed to be satisfactory.

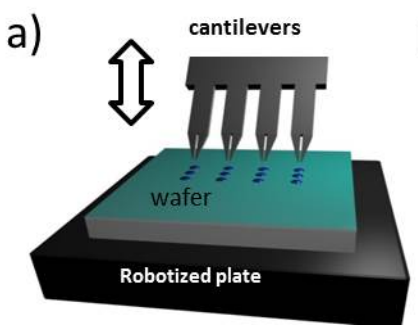

b)

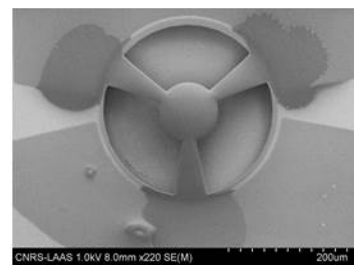

Figure 5: a) Principle of lens deposition using a robotized silicon-cantilever micro-spotter. b) SEM image of membrane surface after deposition of DMSE without surface treatment.
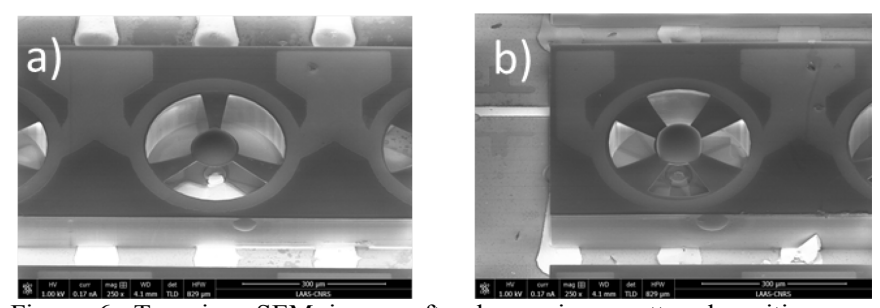

Figure 6: Top-view SEM image after lens micro-spotter deposition on a treated surface using a) DMSE (contact angle $=32^{\circ}$ ) b) polyester (contact angle $=46^{\circ}$ ).

The curvature radius of lenses deposited on these $100-\mu \mathrm{m}-$ diameter circular membranes was measured by confocal microscopy. The corresponding focal length can be simply derived from this measurement, knowing the refractive index and assuming a hemispherical shape [52]. We obtained a focal length of $142.7 \mu \mathrm{m}$ for DMSE (contact angle: $32^{\circ}$ ) and a focal length of $104.1 \mu \mathrm{m}$ for polyester (contact angle: $46^{\circ}$ ). The numerical aperture NA can also be calculated based on the measured curvature radius and focal length. Polyester was 
selected for the rest of the study because a shorter focal length is better suited to obtain a focused beam. Moreover, a higher NA value was obtained $(\mathrm{NA}=0.44)$, which is an advantage for imaging applications. Using this deposition method, the only way to tune the focal length for a given diameter is to change the surface contact angle by modifying the lens material or the surface treatment (fig. 7).

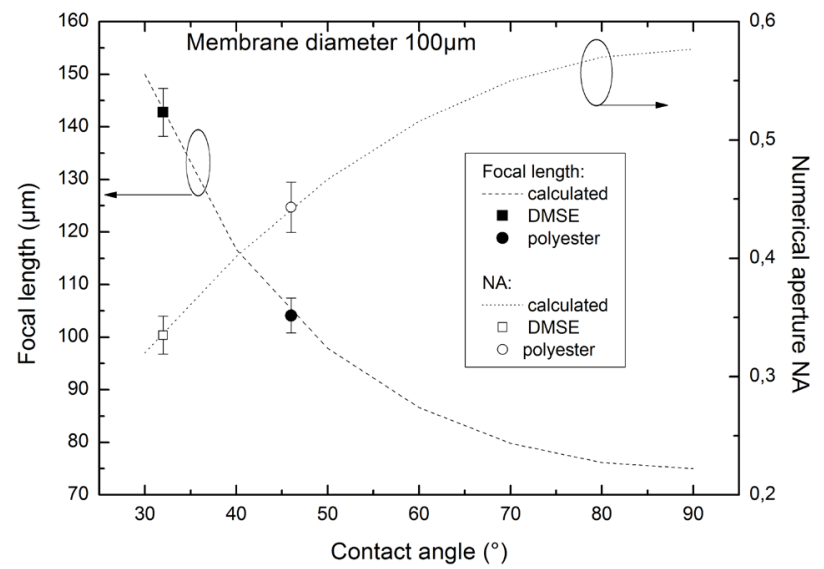

Figure 7: Focal length and numerical aperture of a micro-spotted lens deposited on a $100-\mu$ m-diameter membrane using DMSE (square) and polyester (circle). Error bars represent the standard deviation of the measured values. Calculated values are also plotted as a function of contact angle for a diameter of $100 \mu \mathrm{m}$ (dotted line).

\section{2) Lens deposition using inkjet printing}

Adjusting the lens dimensions during fabrication can be useful to target a different waist size or waist position for a specific application. Under these conditions, modifying the deposition parameters is much easier than changing the lens diameter on the mask, the lens material or the surface treatment applied prior to deposition. For these reasons, we recently tested an inkjet printing technique for our MOEMS technology. This technique indeed makes large changes in lens shape possible by simply modifying the number of printed polymer droplets [43]. Moreover, it should be more suited than our micro-spotting technique for rapid fabrication at the wafer scale. We used a commercial set-up (Altadrop). A polyester material with a viscosity adapted for inkjetting process was used as an ink. Deposition was achieved on membranes submitted to the same wet chemical treatment used for micro-spotted lenses. Under these conditions, the complete coverage of a membrane measuring $100 \mu \mathrm{m}$ in diameter with good lens centering was obtained for a number of printed droplets greater than 4 (fig.8).
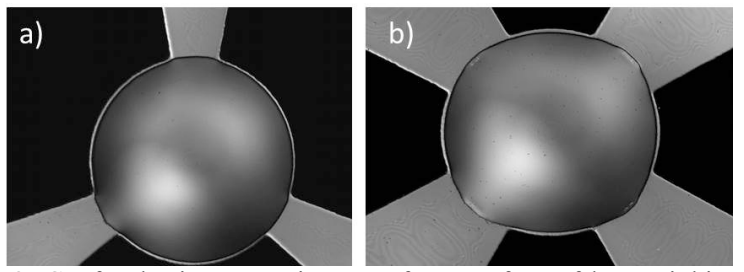

Figure 8: Confocal microscopy images of top surface of lenses inkjetted on circular SU8 membranes measuring $100 \mu \mathrm{m}$ in diameter with a) 3 arms and b) 4 arms (polyester, 8 droplets).
As expected with this technique, the deposited volume increases with the number of drops, leading to a linear decrease in the resulting focal length, calculated using the above-mentioned method, and to a corresponding increase of the numerical aperture (fig. 9). For more than eight droplets, no further variation in volume was observed, and the focal length was constant and close to that obtained using our micro-spotter technique $(\mathrm{f}=102,6 \mu \mathrm{m})$. This behavior is in good agreement with published results concerning inkjet printing on mesas with a fixed diameter [53]. These results confirm that inkjet printing is an appropriate solution for collective fabrication on membranes because it provides an additional tuning parameter for adjusting the lens curvature radius without changing the circular membrane diameter, material properties or the surface energy.

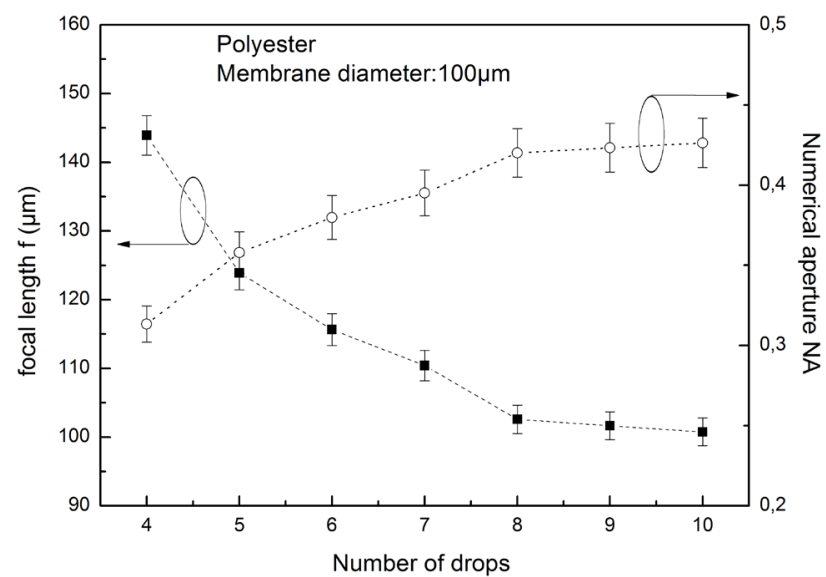

Figure 9: Focal length and numerical aperture of inkjetted microlenses on a $100-\mu \mathrm{m}$-diameter circular membrane as a function of the number of printed droplets (polyester, constant contact angle: $46^{\circ}$ ). Error bars represent the standard deviation of the measured values.

\section{FIRST RESULTS ON MOEMS PERFORMANCES}

MOEMS fabrication was first demonstrated both on glass and on oxidized silicon substrates and more recently on a III-V VCSEL sample. As illustrated in fig. 10, our collective MEMS technology enables many designs, including designs featuring different numbers of actuation arms ( 3 or 4 ) in an array. As shown in fig. 10, lens deposition using a micro-spotter was not observed to be completely reproducible. In some cases, an incomplete coverage of the membrane was indeed observed. Such incomplete coverage occurred because the cantilevers' reservoirs were initially developed for the deposition of droplets of a smaller diameter $(40 \mu \mathrm{m})$. Due to a limited width $(200 \mu \mathrm{m})$, the reservoirs were thus not refilled frequently enough to ensure complete coverage on some membranes. This problem can be easily solved by using larger reservoirs or by using inkjet printing. Taking into account our recent results on inkjet deposition, we recommend the latter option to ensure a rapid and reproducible fabrication. In the following experiment dedicated to characterization, only MOEMS with fully covered membranes were tested. 


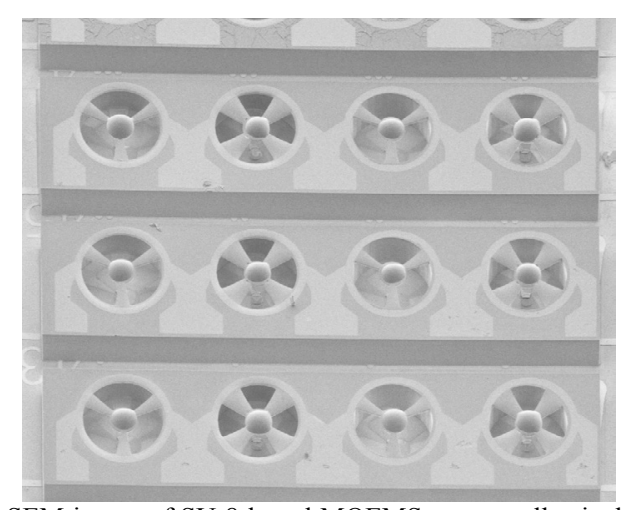

Figure 10: SEM image of SU-8-based MOEMS arrays collectively fabricated on $850-\mathrm{nm}$ VCSELs with a $500-\mu \mathrm{m}$ pitch.

\section{A. MOEMS mechanical behavior}

Optical interferometry under probes was used to characterize the MOEMS behavior before lens deposition. We verified that the membranes remained flat under actuation, with the radius of curvature remaining equal to $2 \mathrm{~mm}$. Significant vertical displacement of the membrane was measured as a function of applied power (fig. 11).

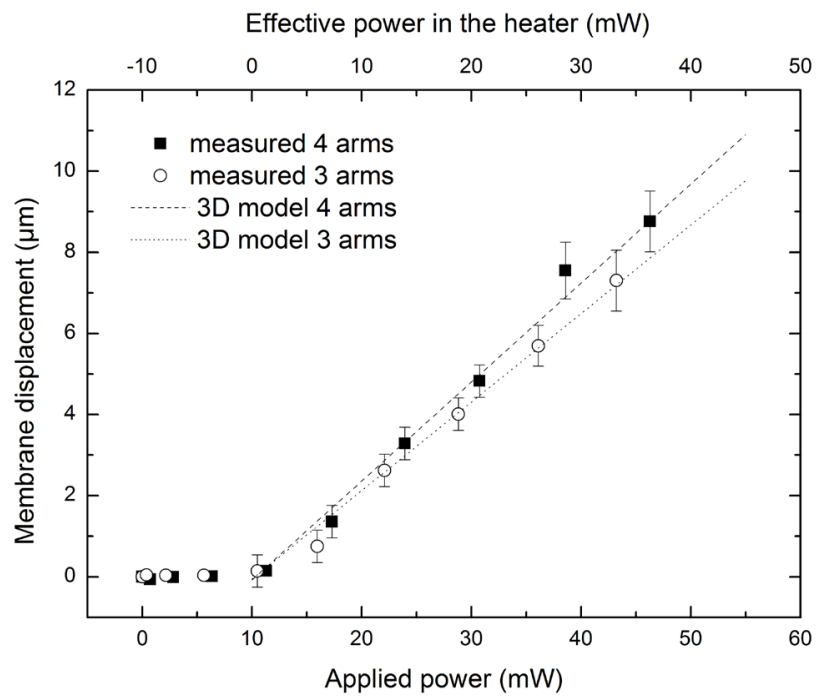

Figure 11: Membrane axial displacement measured as a function of applied power to the MOEMS for 3-arm (open circles) and 4-arm (squares) configurations. Error bars represent standard deviation of the measured values. Simulated behavior is also plotted as a dotted line as a function of the effective power dissipated in the heater.

In the linear part of the variation curve, the slope was as high as $+0.25 \mu \mathrm{m} / \mathrm{mW}$ at applied voltages lower than $3 \mathrm{~V}$. These results confirm the interest in exploiting the high thermal dilatation of SU-8, as previously discussed. Similar trends were observed regardless of the number of MOEMS arms (3 or 4), with a slightly better efficiency for the 4 arm configuration. It is worth noting that the effective power dissipated in the actuation arms was lower than the indicated value because electrical losses due to the resistance between the probes and the titanium electrode were estimated to be equal to $10 \mathrm{~mW}$. Taking into account this parasitic effect, we recently observed good agreement between measurements using four probes (instead of two) and theoretical MEMS behavior indicated by $3 \mathrm{D}$ electro-thermo-mechanical modeling
(COMSOL). The vertical displacement yielded by 3D modeling is plotted as a function of the effective power dissipated in the heater in fig. 11 to illustrate the good agreement with the experimental data for both cases. Further details on this study can be found elsewhere [54]. Additional studies on MEMS cycling and reliability are now underway to estimate thermal actuation hysteresis observed for effective power greater than $30 \mathrm{~mW}$ corresponding to an actuation temperature of $150^{\circ} \mathrm{C}$.

\section{B. MOEMS optical behavior}

SU-8 MOEMS arrays featuring micro-spotted polyester microlenses were fabricated on electrically driven VCSELs emitting at $850 \mathrm{~nm}$. Unfortunately, these devices were not single-mode as initially envisioned because the size of the emission zone was larger than expected due to a fabrication issue $(12 \mu \mathrm{m}$ instead of $3 \mu \mathrm{m})$. As a result, the devices emitted a highly multimodal transverse beam with a higher divergence than that of the Gaussian beam we used for our MOEMS design $\left(18^{\circ}\right.$ instead of $\left.12^{\circ}\right)$. Nevertheless, we tried to estimate the change in the laser spot due to the presence of an integrated tunable lens by measuring the spot size at different positions along the optical axis with a movable camera placed above devices tested under probes. As shown in fig. 12, laser beam focusing was clearly observed at distances between 300 and $400 \mu \mathrm{m}$ from the membrane surface, with an estimated FWHM of $14 \mu \mathrm{m}$. Although a direct comparison to modeling is not possible, a similar focusing position was indicated by our calculations, assuming an initial Gaussian beam with a similar emission zone and divergence. To estimate the effect of actuation on the focused spot position, we measured the variation of its maximal intensity along the axis near the focus position. This measurement was performed with and without MOEMS actuation. To remain in a linear regime and to ensure well reproducible behavior, the MOEMS maximal driving power was limited to $28 \mathrm{~mW}$, corresponding to an axial displacement of $+4 \mu \mathrm{m}$. Under these conditions, we observed a shift in the maximal spot intensity of $-35 \mu \mathrm{m}$ (fig. 13a); this value is of course lower than the one expected for a singlemode VCSEL $(>70 \mu \mathrm{m})$. Nevertheless, the value is in quite good agreement with our optical modeling results, assuming the same numerical aperture of the source and an axial shift of $+4 \mu \mathrm{m}$ (fig. $3 \mathrm{~b}$ ). These preliminary results are very promising because they demonstrate, for the first time, the feasibility of integrating polymer-based movable lenses arrays on laser arrays.

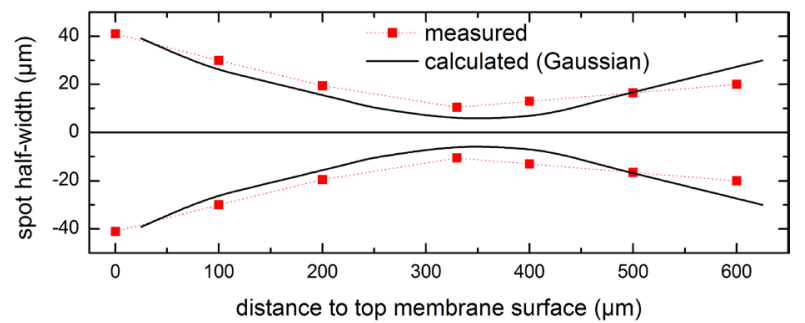

Figure 12: Measurement of VCSEL spot width as a function of the axial position (red squares, dotted line) and comparison with calculated beam profile assuming Gaussian beam propagation (black line). 


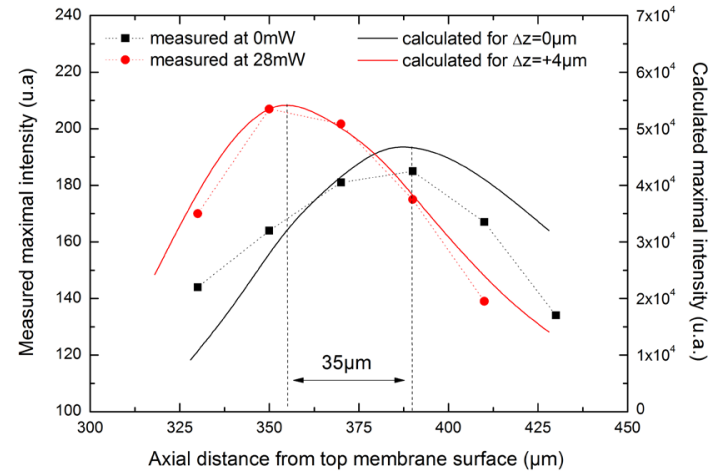

Figure 13: Maximal spot intensity measured as a function of axial position under MOEMS actuation at $0 \mathrm{~mW}$ (black square) and $28 \mathrm{~mW}$ (red circles) and comparison with calculated values for axial shifts of $0 \mu \mathrm{m}$ (black line) and 4 $\mu \mathrm{m}$ (red line).

\section{CONCLUSIONS AND FUTURE WORK}

We presented our recent work on polymer-based MOEMS fabrication to achieve tunable micro-optics on VCSEL arrays. After discussing the relative interest in polymers compared to that in silicon-based materials for integrated optical MEMS, we detailed the simple and collective SU-8-based technology we developed for VCSEL beam dynamic focusing. We highlighted in particular two critical fabrication issues we recently solved. The first one concerns the uniform fabrication of thick high-aspect-ratio MOEMS on small III-V samples. We proposed to use an alternative to spin coating that we call "soft thermal printing" to ensure uniform SU-8 deposition regardless of sample shape, properties or size. The second addresses the precise fabrication of a microlens and its alignment with the VCSEL source. We experimentally evaluated two liquid dispensing methods and demonstrated that they are both compatible with self-alignment on a suspended SU-8 membrane. As a result, the alignment precision is fixed by photolithography $(2 \mu \mathrm{m})$ and thus fulfills all requirements regarding lateral tolerances. Our MOEMS exhibited large mechanical displacements under low electrothermal actuation voltages and was integrated on multimode VCSELs arrays using micro-spotted lenses. Beam focusing was experimentally observed at a distance in good agreement with that yielded by optical modeling, taking into account the characteristics of the laser beam. Moreover, we observed a shift in focus position of $35 \mu \mathrm{m}$ under actuation. To fully validate our approach, we are currently fabricating MOEMS on single-mode VCSELs for which we expect larger optical scans under actuation. Moreover, high-quality Gaussian beams are most often required, especially for instrumentation and biosensing applications. Finally, our latest advances in soft thermal printing and inkjet printing are being implemented in this new set of tests to further improve air gap control and lens positioning and to make the adjustment of the microlens focal length during fabrication possible. To conclude, SU-8-based optical MEMS devices have become very attractive for VCSEL beam focusing and they could also be exploited for other beam shaping functions, such as off-axis beam steering. Looking further ahead, SU-8 could also replace dielectric materials for the low-cost fabrication of spectrally tunable MEMS-VCSELs, although some issues must still be resolved, such as the fabrication of highly reflective mirrors on a polymeric membrane. Nevertheless, the fabrication of an electrically-tunable organic microcavity source was reported recently [55], illustrating the growing interest in organic MOEMS.

\section{Acknowledgment}

The authors gratefully acknowledge Fabien Mesnilgrente and Véronique Conédéra for their contribution in inkjet printing and Sandrine Assié-Souleille for her support for MEMS actuation measurements.

\section{REFERENCES}

[1] K. Iga, "Vertical-Cavity Surface-Emitting Laser: Its Conception and Evolution" Japanese Journal of Applied Physics Vol. 47, No. 1 pp. 1-10, 2008

[2] A.Larsson, "Advances in VCSELs for Communication and Sensing," Selected Topics in Quantum Electronics, IEEE Journal of, vol.17, no.6, pp.1552,1567, Nov.-Dec. 2011.

[3] R. Michalzik "VCSELs: A Research Review" in Fundamentals, Technology and Applications of Vertical-Cavity Surface-Emitting Lasers. Springer, pp. 1-17, 2013.

[4] E. Thrush, O. Levi, W. Ha, G. Carey, L.J. Cook, J. Deich, S.J. Smith, W.E. Moerner, J.S., Jr. Harris, "Integrated semiconductor vertical-cavity surface-emitting lasers and PIN photodetectors for biomedical fluorescence sensing," IEEE Journal of Quantum Electronics 40,5., pp. 491-498, 2004.

[5] J. Perchoux and T.Bosch, "Multimode VCSELs for Self-Mixing Velocity Measurements," Sensors, 2007 IEEE, vol., no., pp.419-422, 28$31,2007$.

[6] A. Kroner, I. Kardosh, F. Rinaldi, R. Michalzik, "Towards VCSELbased integrated optical traps for biomedical applications," Electronics Letters, vol.42, no.2, pp. 93-94, 2006.

[7] L.M. Lechuga, J. Tamayo, M. Álvarez, L.G. Carrascosa, A. Yufera, R. Roldán, E. Peralías, A. Rueda, J.A. Plaza, K. Zinoviev and C Domínguez, A. Zaballos, M. Moreno and C. Martínez-A, D. Wenn, N. Harris, C. Bringer, V. Bardinal, T. Camps, C. Vergnenègre and C. Fontaine, V. Díaz and A. Bernad. "A Highly sensitive Microsystem Based on nanomechanical biosensors for genomics applications" Sensors and Actuators B, Vol.118, $\mathrm{N}^{\circ} 1-2, \mathrm{pp} .2-10,2006$.

[8] D. Heinis, C. Gorecki, C. Bringer, V. Bardinal, T. Camps, J.-B. Doucet, P. Dubreuil and C.Fontaine "Miniaturized Scanning Near-Field Microscope Sensor Based on Optical Feedback inside a Single-Mode Oxide-Confined Vertical-Cavity Surface-Emitting Laser" Jpn. J. Appl. Phys. Vol 42 pp. L1469-L1471, Part2, N¹2A, 2003.

[9] Koyama, F., Kinoshita, S., and Iga, K. 'Room-temperature continuous wave lasing characteristics of a GaAs vertical cavity surface-emitting laser'. Applied physics letters, 55(3), 221-222. 1989

[10] Jewell, J. L., Lee, Y. H., Walker, S., Scherer, A., Harbison, J. P., Florez, L. T., and McCall, S. L. (1989). Low-threshold electrically pumped vertical-cavity surface-emitting microlasers. Electronics Letters, 25(17), 1123-1124.

[11] Huffaker, D. L., Deppe, D. G., Kumar, K., and Rogers, T. J. (1994). Native-oxide defined ring contact for low threshold vertical-cavity lasers. Applied Physics Letters, 65(1), 97-99.

[12] Ortsiefer, M.; Hofmann, W.; Rönneberg, E.; Boletti, A.; Gatto, A.; Boffi, P.; Rosskopf, J.; Shau, R.; Neumeyr, C.; Böhm, G.; Martinelli, M.; Amann, M.-C. (2008). High speed $1.3 \mu \mathrm{m}$ VCSELs for $12.5 \mathrm{Gbit} / \mathrm{s}$ optical interconnects. Electronics Letters, 44(16), 974-975.

[13] Danner, A. J., Kim, T. S., and Choquette, K. D. (2005). Single fundamental mode photonic crystal vertical cavity laser with improved output power. Electronics Letters, 41(6), 325-326.

[14] M. C. Y. Huang, Y. Zhou, and C. J. Chang-Hasnain, "Single mode highcontrast subwavelength grating vertical cavity surface emitting lasers" Appl. Phys. Lett. 92, 171108, 10.1063/1.2917447,2008.

[15] C.J. Chang-Hasnain, "Tunable VCSEL," IEEE Journal of Selected Topics in Quantum Electronics, vol.6, no.6, pp.978-987,„2000.

[16] Wu, M. S., Vail, E. C., Li, G. S., Yuen, W., and Chang-Hasnain, C. J.. Widely and continuously tunable micromachined resonant cavity 
detector with wavelength tracking. Photonics Technology Letters, IEEE, 8(1), 98-100. 1996

[17] Nakahama, M.; Sano, H.; Inoue, S.; Sakaguchi, T.; Matsutani, A.; Koyama, F., "Tuning Characteristics of Monolithic MEMS VCSELs With Oxide Anti-Reflection Layer," Photonics Technology Letters, IEEE, vol.25, no.18, pp.1747,1750, Sept.15, 2013

[18] M. Maute, F. Riemenschneider, G. Bohm, H. Halbritter, M. Ortsiefer, R P. Shau, Meissner, M.-C Amann,.; "Micro-mechanically tunable long wavelength VCSEL with buried tunnel junction" Electronics Letters 1 Volume: 40 Issue:7,2004.

[19] B. Kögel, A. Abbaszadehbanaeiyan, P. Westbergh, A. Haglund, J. Gustavsson, J. Bengtsson, E. Haglund, H. Frederiksen, P. Debernardi, A. Larsson "Integrated tunable VCSELs with simple MEMS technology," Semiconductor Laser Conference,ISLC., 2010 22nd IEEE International pp.1-2, 26-30, 2010.

[20] C. Gierl, T. Gruendl, P. Debernardi, K. Zogal, C. Grasse, H. Davani, G. Böhm, S. Jatta, F. Küppers, P. Meißner, and M. Amann, "Surface micromachined tunable $1.55 \mu \mathrm{m}$-VCSEL with $102 \mathrm{~nm}$ continuous single-mode tuning," Opt. Express 19, 17336-17343, 2011.

[21] Jayaraman, V.; Cole, G.D.; Robertson, M.; Burgner, C.; John, D.; Uddin, A.; Cable, A.: 'Rapidly swept, ultra-widely-tunable $1060 \mathrm{~nm}$ MEMS-VCSELs', Electronics Letters,, 48, (21), p. 1331-1333. 2012

[22] V. Bardinal, T. Camps, B. Reig, D. Barat, E. Daran, and J. B. Doucet "Collective micro-optics technologies for VCSEL photonic integration", Advances in Optical Technologies, vol. 2011, 609643, 2011.

[23] L. Fan, M. C. Wu, H. C. Lee, and P. Grodzinski "Dynamic Beam Switching of Vertical-Cavity Surface-Emitting Lasers with Integrated Optical Beam Routers" IEEE Photon. Technol. Lett., Vol. 9, nº. 4, 1997.

[24] M.C. Wu, L.-Y. Lin, S.-S. Lee and K.S.J. Pister "Micromachined freespace integrated micro-optics" Sensors and Actuators A: Physical Volume 50, Issues 1-2, Pages 127-134,1995.

[25] A. Tuantranont, V. M. Bright, J. Zhang, W. Zhang, J. A. Neff and Y. C. Lee "Optical beam steering using MEMS-controllable microlens array" Sensors and Actuators A: Physical, Volume 91, Issue 3, 2001.

[26] K. Ishikawa, J. Zhang, A. Tuantranont, V.M. Bright and Y.-C. Lee "An integrated micro-optical system for VCSEL-to-fiber active alignment" Sensors and actuators A 103 109-115, 2003.

[27] Jo K W, Kim M S, Lee J H, Kim E K, and Park K H "Optical characteristics of a self-aligned microlens fabricated on the sidewall of a $45^{\circ}$ angled optical fiber" IEEE Photonics Technology Letters, Vol. 16, No. 1, pp. 138-140, 2004.

[28] K. Hedsten, J. Melin, J. Bengtsson, P. Modh, D. Karlén, B. Löfving, R. Nilsson, H.Rödjegård, K. Persson, P. Enoksson, F. Nikolajeff, G. Andersson "MEMS-based VCSEL beam steering using replicated polymer diffractive lens" Sensors and Actuators A: Physical, Volume 142, Issue 1, pp. 336-345, 2008.

[29] N. Quack, J.B. Chou, M.C. Wu, "Self-aligned VCSEL-microlens scanner with large scan range," Micro Electro Mechanical Systems,MEMS., 2012 IEEE 25th International Conference on, pp.656659, Jan. 29 2012-Feb. 2, 2012.

[30] B Reig, T Camps, V Bardinal, D Bourrier, E Daran, J B Doucet, J Launay and J Y Fourniols "Fabrication of polymer-based optical microsystem arrays suited for the active focusing of vertical laser diodes "J. Micromech. Microeng. 22 065006, 2012.

[31] Reig, B.; Bardinal, V.; Camps, T.; Doucet, J.; Daran, E., "A miniaturized VCSEL-based system for optical sensing in a microfluidic channel," Sensors, IEEE, vol., no., pp.1,4, 28-31 Oct. 2012,2012.

[32] H. Lorenz, M. Despont, N. Fahrni, J. Brugger, P. Vettiger, P. Renaud, "High aspect-ratio, ultrathick, negative-tone near-UV photoresist and its applications for MEMS", Sensors and Actuators A 64, 33-39 (1998).

[33] R. Feng, R. J. Farris "The characterization of thermal and elastic constants for an epoxy photoresist SU-8 coating" Journal of Material Science 37, 4793 - 4799, 2002.

[34] Namazu, T., Inoue, S., Takio, K., Fujita, T., Maenaka, K., and Koterazawa, K.. "Visco-elastic properties of micron-thick SU-8 polymers measured by two different types of uniaxial tensile tests". In Micro Electro Mechanical Systems, 2005. MEMS 2005. 18th IEEE International Conference on (pp. 447-450). IEEE. 2005.

[35] Fujita, T., Maenaka, K., and Takayama, Y.. "Dual-axis MEMS mirror for large deflection-angle using SU-8 soft torsion beam. Sensors and Actuators A: Physical, 121(1), 16-21, 2005.

[36] Wang, L., Sipe, D. M., Xu, Y., and Lin, Q. "A MEMS thermal biosensor for metabolic monitoring applications". Microelectromechanical Systems, Journal of, 17(2), 318-327. 2008.
[37] Seidemann, V., Bütefisch, S., and Büttgenbach, S. «Fabrication and investigation of in-plane compliant SU8 structures for MEMS and their application to micro valves and micro grippers". Sensors and Actuators A: Physical, 97, 457-461. 2002.

[38] Park, S. B., Chung, S., Makhar, S., Ackler, H., and Lin, P. (2005, January). "Structural reliability of SU-8 material for MEMS application." In ASME 2005 International Mechanical Engineering Congress and Exposition (pp. 251-258). American Society of Mechanical Engineers.2005.

[39] B. Reig, V. Bardinal, J.-B. Doucet, E. Daran, T. Camps, M. Aufray, A. Lamure and C. Tendero. "Study of SU-8 reliability in wet thermal ambient for application to polymer micro-optics on VCSELs" Jpn. J. Appl. Phys. 5308 MC03, 2014.

[40] B Bêche, N Pelletier, E Gaviot, J Zyss, «Single-mode TE00-TM00 optical waveguides on SU-8 polymer ", Optics Communications, Volume 230, Issues 1-3, Pages 91-94, 15 January 2004

[41] Beche, B., "Integrated photonics devices on Su8 organic materials". Int. J. of Phys. Sc, 5, 612-618. 2010.

[42] Hsieh, J., Weng, C. J., Yin, H. L., Lin, H. H., and Chou, H. Y. "Realization and characterization of SU-8 micro cylindrical lenses for in-plane micro optical systems". Microsystem technologies, 11(6), 429437, 2005

[43] D.J. Hayes, M.E. Grove, D.B. Wallace, T.Chen, W.R. Cox, "Ink-jet printing in the manufacturing of electronics, photonics, and displays" Proceedings of SPIE, vol. 4809 Nanoscale Optics and Application, pp.94-99, 2002.

[44] A. Nallani, T. Chen, J.-B. Lee, D. Hayes and D. Wallace, "Wafer level optoelectronic device packaging using MEMS“, Proc. SPIE 5836, 116, 2005

[45] V. Bardinal, B. Reig, T. Camps, C. Levallois, E. Daran, C. Vergnenègre, T. Leïchlé, G. Almuneau, and J.-B. Doucet. "Spotted Custom Lenses to Tailor the Divergence of Vertical-Cavity Surface-Emitting Lasers IEEE Photon. Technol. Lett., Vol.22, N’21, pp.1592-1594, 2010.

[46] B. Reig, V. Bardinal, T. Camps, Y. G. Boucher, C. Levallois, J. B. Doucet, D. Bourrier, E. Daran, J. Launay " Polymer tunable microlens arrays suitable for VCSEL beam control", Proc. SPIE 8428, MicroOptics 2012, 84280N, 2012.

[47] L. Campagnolo, M. Nikolić, J. Perchoux, Y.L. Lim, K. Bertling, K.Loubière, L. Prat, A.D. Rakić and T. Bosch, " Flow profile measurement in microchannel using the optical feedback interferometry sensing technique ", Microfluidics and Nanofluidics, SpringerVerlag,,1-7, 2012

[48] Ceyssens F and Puers R., "Creating multi-layered structures with freestanding parts in SU-8", Journal of Micromechanics and Microengineering, Vol. 16, S19-S23, 2006.

[49] P. Abgrall, C. Lattes, V. Conédéra, X. Dollat, S. Colin and A.M. Gué, "A novel fabrication method of flexible and monolithic 3D microfluidic structures using lamination of SU-8 films" J. Micromech. Microeng. 16 $113-21,2006$

[50] S. Abada; B. Reig; E. Daran; JB Doucet; T. Camps; S. Charlot; V. Bardinal; "Uniform fabrication of thick SU-8 patterns on small-sized wafers for micro-optics applications", Proc. SPIE 9130, Micro-Optics 2014, 91300R, May 2, 2014

[51] C. Levallois, V. Bardinal, T. Camps, T. Leïchlé, E. Daran, J.B. Doucet and C. Vergnenègre, "VCSEL collimation using self-aligned integrated polymer microlenses", Proc. SPIE, 699269920 W, 2008.

[52] V. Bardinal, E. Daran, T. Leïchlé, C. Vergnenègre, C. Levallois, T. Camps, V. Conedera, J. Doucet, F. Carcenac, H. Ottevaere, and H. Thienpont, "Fabrication and characterization of microlens arrays using a cantilever-based spotter," Opt. Express 15, 6900-6907, 2007.

[53] J. Y. Kim, C. Martin-Olmos, N. S. Baek and J. Brugger, "Simple and easily controllable parabolic-shaped microlenses printed on polymeric mesas", J. Mater. Chem. C, 1, 2152-2157, 2013.

[54] S. Abada; T. Camps; B. Reig; JB Doucet; E. Daran; V. Bardinal; "3D optimization of a polymer MOEMS for active focusing of VCSEL beam" Proc. SPIE 9130, Micro-Optics 2014, 913003, May 2, 2014

[55] Wang, A; Chang, W.; Murarka, A; Lang, J.H.; Bulovic, V., "Transferprinted composite membranes for electrically-tunable organic optical microcavities," Micro Electro Mechanical Systems (MEMS), 2014 IEEE 27th International Conference on, pp.1217,1220, 26-30 Jan., 2014. 